\title{
Quantifying coral morphology
}

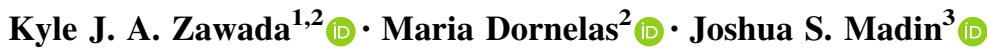

Received: 20 February 2019/Accepted: 2 July 2019/Published online: 2 August 2019

(C) The Author(s) 2019

\begin{abstract}
Coral morphology has important implications across scales, from differences in physiology, to the environments they are found, through to their role as ecosystem engineers. However, quantifying morphology across taxa is difficult, and so morphological variation is typically captured via coarse growth form categories (e.g. arborescent and massive). In this study, we develop an approach for quantifying coral morphology by identifying continuous three-dimensional shape variables. To do so, we contrast six variables estimated from 152 laser scans of coral colonies that ranged across seven growth form categories and three orders of magnitude of size. We found that $88 \%$ of the variation in shape was captured by two principal components. The main component was variation in volume compactness ( $c f$. convexity), and the second component was a trade-off between surface complexity and topheaviness. Variation in volume compactness also limited variation along the second axis, where surface complexity and top-heaviness ranged more freely when compactness was low. Traditional growth form categories occupied
\end{abstract}

Topic Editor: Morgan S. Pratchett

Electronic supplementary material The online version of this article (https://doi.org/10.1007/s00338-019-01842-4) contains supplementary material, which is available to authorized users.

Kyle J. A. Zawada

kz28@st-andrews.ac.uk

1 Centre for Biological Diversity, Scottish Oceans Institute, University of St. Andrews, Scotland KY16 9TH, UK

2 Department of Biological Sciences, Macquarie University, Sydney, NSW 2109, Australia

3 Hawai ' $i$ Institute of Marine Biology, University of Hawai ' $i$ at Mānoa, Kaneohe, HI, USA distinct regions within this morphospace; however, these regions overlapped due to scaling of shape variables with colony size. Nonetheless, with four of the shape variables we were able to predict traditional growth form categories with 70 to $95 \%$ accuracy, suggesting that the continuous variables captured most of the qualitative variations implied by these growth forms. Distilling coral morphology into continuous variables that capture shape variation will allow for better tests of the mechanisms that govern coral biology, ecology and ecosystem services such as reef building and provision of habitat.

Keywords Functional morphology $\cdot$ Scleractinia $\cdot 3 D$ scanning · Shape analysis - Traits · Growth form

\section{Introduction}

The shape and size of organisms determines how they interact with the physical environment and with other organisms (Denny 1993; Vogel 1996). This is especially true for sessile colonial organisms, where variation in morphology has been linked to a range of biological and ecological processes (Jackson 1977, 1979). For example, growing upwards from the benthos reduces benthic competition, while growing laterally reduces whole colony mortality by spreading risk (Jackson 1979). Despite the fundamental importance of a colony's morphology, there is no general framework for capturing morphological variation. Instead, scientists tend to lump individuals and species into discrete growth form categories [corals: (Veron 2000); bryozoans: (Bishop 1989); bacteria: (Shapiro 1995)] or use continuous metrics that cannot partition the effect of size and shape [e.g. surface area-to-volume ratio; (Naumann et al. 2009)]. Developing a quantitative framework is 
challenging for colonial organisms because they have geometrically complex forms, high intraspecific and interspecific variation in shape, and lack readily identifiable landmarks for comparative analysis. However, new technologies such as CT and laser scanning make it possible to accurately capture the diversity of shapes exhibited by colonial organisms (Lavy et al. 2015; House et al. 2018). Here, using reef corals as a study system, we develop a morphological schema using quantitative, threedimensional shape variables.

Scleractinian corals are a prime example of colonial organisms whose morphology directly dictates life history strategies (Jackson 1979), demographic rates (Madin et al. 2014; Álvarez-Noriega et al. 2016; Dornelas et al. 2017) and provisioning of habitat for other taxa (Bell and Galzin 1984; Graham and Nash 2013; Richardson et al. 2017b). The aragonite skeleton that Scleractinian corals secrete as they grow provides support and shape; however, most of the live biomass is associated with the surface (Johannes and Wiebe 1970; Hoegh-Guldberg 1988). These characteristics have consequences for vital processes such as growth and survival, where higher surface area-to-volume ratios allow more biomass per unit investment in skeleton, but may increase the risk of partial colony mortality (Lirman 2000), dislodgement (Madin 2005), and susceptibility to thermal bleaching (Baird and Marshall 2002). In parallel, coral structures provide direct habitat for many taxa and can act as predator refuge for both adult and juvenile fishes (Friedlander and Parrish 1998; Kerry and Bellwood 2012). At the habitat scale, variation in the morphology of each colony in an assemblage contributes to the overall structural complexity of the habitat (Richardson et al. 2017a), which has been linked to ecosystem properties, such as microhabitat availability (Graham and Nash 2013) which in turn influences community structure (Almany 2004; Nash et al. 2014), and larval recruitment (Hata et al. 2017). Furthermore, variation in colony shape influences the persistence of colony skeleton following mortality and reef matrix building and infilling processes (Rasser and Riegl 2002; Glynn and Manzello 2015). Yet, despite the importance of morphology for the functioning of both the corals themselves and coral reef ecosystems, quantitative studies of coral morphology are sparse, presumably because of difficulties in measuring and dealing with the geometric complexity of coral forms.

Scleractinian corals exhibit high levels of variation in morphology within and among taxa. They vary from simple shapes, such as encrusting or hemispherical colonies, to tree-like branching shapes. There are also varying degrees of morphological plasticity within species driven by interactions with local environmental conditions (Foster 1979), though some variation among species is genetically constrained (Filatov et al. 2013). Additional phenomena such as partial mortality (Meesters et al. 1996), colony fragmentation (Karlson 1986) and indeterminate growth (Sebens 1987) add to the complexity and observed variation in morphology from colony to colony, even within species and conspecifics. Corals need access to free-flowing water for filter feeding, and light for photosynthesis, both of which are linked to morphological variation (Kaandorp et al. 1996; Hoogenboom et al. 2008). Additionally, competition for space results in many colonies growing up from the substrate to increase standing biomass without needing to continuously colonise new substrate (Jackson 1977). However, many sessile colonial organisms within marine environments are subjected to hydrodynamic forces that can dislodge entire colonies if they grow too far away from the substrate, restricting the range of available morphologies (Koehl 1999). Taken together, coral colonies exhibit multiple morphological trade-offs that result in the vast array of observed variation in morphology (Chappell 1980; Kaandorp et al. 1996).

Scleractinian corals are typically categorised into growth forms based on coarse morphological similarities. Growth forms are useful for species identification and monitoring changes in assemblage structure, but do not adequately capture geometric complexity or intraspecific variation in shape. Phenotypic plasticity is common among coral species, where the same species can exhibit different growth forms in different environments (Veron 2002). Despite these limitations, growth form is a useful metric because life processes can differ significantly among categories. For example, growth form is a good predictor of competitive ability (Connell et al. 2004; Hoogenboom et al. 2008) and zonation patterns (Chappell 1980; Done 2011). Growth form and size affect demographic rates including fecundity (Álvarez-Noriega et al. 2016), growth (Dornelas et al. 2017) and background mortality (Madin et al. 2014). While these results highlight differences between growth forms across a range of processes, they are unable to directly assess process-based hypotheses for the observed differences, nor can the results be generalised to other growth forms or taxa with similar morphological adaptations but different overall morphology (e.g. sponges, hydrozoans, algae, plants, etc.). As such, recent studies have begun to explore techniques for quantifying and comparing the three-dimensional shape of corals (Bythell et al. 2001; Filatov et al. 2010, 2013; Lavy et al. 2015; Reichert et al. 2017; House et al. 2018). We build on this work to develop a quantitative schema for coral morphology via variables that capture shape variation.

Many processes in corals have size-dependent relationships (Madin et al. 2014; Dornelas et al. 2017); however, these relationships change depending on growth form, suggesting that colony shape is also important. Quantitative variables that attempt to explain these differences should 
therefore aim to be causally linked to processes and partition the effects of shape and size separately. Morphology has previously been quantified using variables such as corallite area and branch spacing that may have implications locally within a colony or at the polyp scale (Bruno and Edmunds 1997; Shaish et al. 2007). Surface area-tovolume ratios are an example of whole colony information with expected causal links to processes (Hoegh-Guldberg 1988). However, surface area-to-volume ratio can change with either differences in shape or size or both, making the effects of shape and size on other processes difficult to disentangle when using this metric. Recent work within the genus Madracis has quantified coral branching patterns and growth processes using 3D imaging (Kaandorp et al. 1996; Filatov et al. 2010); however, measuring whole colony morphology across a broad taxonomic and morphological scale along multiple axes of variation simultaneously has been limited. By measuring multiple, size-independent variables across a wide range of morphological variation, trade-offs and broader patterns become clearer, including how shape changes as colonies get larger.

Variables that can capture how a colony is spatially distributed in the environment should capture functionally relevant axes of morphological variation. For example, variables that measure colony volume compactness may act as a good indicator of "branchiness" or how sturdy a colony is, capturing a continuous gradient from massive to arborescent colonies. Variation in compactness may therefore covary with processes such as growth rates, fragmentation and habitat provision (Gladfelter et al. 1978; Lirman 2000; Alvarez-Filip et al. 2011). Two variables that can capture volume compactness are convexity and sphericity, where convexity captures the ratio of the objects volume to the volume immediately surrounding it, and sphericity which captures how close an objects shape is to a sphere, which is the most compact shape possible in three dimensions.

Another axis of variation is how the surface area of a colony is distributed in space, which should capture a gradient from flatter, less convoluted surfaces to colonies with highly complex and convoluted surfaces. Variation in surface complexity may capture a functional trade-off axis between biomass packing (e.g. having more biomass for a given area of space) and decreased intra-colony competition for resources (e.g. increased light per unit biomass when surface area is spread out) (Hoogenboom et al. 2008; Wangpraseurt et al. 2012). Surface complexity can be captured with two shape variables, fractal dimension, which captures how surface area fills space at different scales and is an estimate of spatial complexity, and packing, which captures how much of an object's surface area is situated internally versus externally in relation to its immediate environment.
The previous four shape variables are all rotationally and size invariant (i.e. the orientation or size of colony meshes has no bearing on the resulting value). However, a distinguishing feature of shape in competing benthic organisms is how volume and surface area are distributed vertically above the substrate, or "top-heaviness". For instance, a tabular coral colony has volume and surface area distributed further away from the substrate than a hemispherical colony that is "bottom heavy". To capture this feature, we used first moments of volume ( $\left.V_{\mathrm{VOL}}\right)$ and surface area $\left(V_{\text {AREA }}\right)$, which are the sums of the products of volume and area, respectively, with their vertical distance from the colony attachment point. This axis can be expected to covary with processes such as whole colony dislodgment, benthic competition strategy and microhabitat diversity (Jackson 1979; Kerry and Bellwood 2012; Madin et al. 2014).

Morphology is important for corals and the ecosystems they build, but a comprehensive suite of quantitative traits for developing explanatory and generalised models for these processes has yet to be formalised. By measuring these axes of morphological variation, we can place coral colonies along multiple functional axes, moving from a subjective, categorical framework towards a quantitative, functional trait-based one. The aim of this study was to measure a set of morphological variables that capture biologically relevant axes of variation in corals. To achieve this aim, we first derived six morphological variables and measured them across a broad diversity of colony shapes and a wide range of sizes via high-resolution $3 \mathrm{D}$ laser scanning. We then asked: (i) how do corals occupy continuous morphological space? (ii) where do growth form classifications sit within this space? (iii) how does the shape of growth forms covary with size? and (iv) do continuous variables capture the subjective information encoded in growth forms? We show that the variables outlined in this study can place growth forms on three continuous axes of variation and provide a more precise, mechanistic toolkit for ongoing research.

\section{Materials and methods}

\section{Data collection}

Colony skeletons from coral collections at the Natural History Museum in London (UK), the Bell Pettigrew Museum at the University of St Andrews (UK) and the Museum of Tropical Queensland (Australia) were scanned using an optical laser scanner (EXAScan, Creaform.inc) and proprietary processing software (VXElements 5, Creaform.inc) to digitize their three-dimensional morphology. The scanner software builds a triangulated mesh 
during data collection to output a range of mesh file types without the need for further processing. Colonies were selected for scanning to capture a broad range of shapes and sizes across a diversity of traditional coral growth form classifications [arborescent, corymbose, digitate, laminar, massive, sub-massive or tabular; (Veron 2000)]. Colonies were also selected to be mostly intact with few breakages; however, in some cases a minor amount of damage was present. The final data set included seven growth forms, 38 species and ranged in size from 5.3 to $9242.5 \mathrm{~cm}^{3}$ (for further details, see Table S1). Specimens were from a broad range of environments and locations making the results of this study more variable than if specimens were collected from the same environment. The use of coral skeletons instead of live colonies means that some surface properties at the microstructural scale are likely to be different for colonies in situ, but is appropriate to capture whole colony morphology.

Scanning was conducted with a standard resolution of $0.5 \mathrm{~mm}^{2}$ but needed to be decreased to $1 \mathrm{~mm}^{2}$ for several large or complex colonies due to computational constraints. All colony scans were orientated with the $z$-axis aligned with the colony's likely upward orientation when on the reef. Each scan consisted of a digital 3D mesh that was comprised of a single contiguous surface of connected triangles. In many cases, non-coral substrate was also included in the scan data and was removed prior to analysis. Meshes were rejected if the final mesh deviated in shape from the actual specimen due to issues associated with interpolating missing scan data. Growth form categories were selected if there were at least five observations of sufficient quality. The final dataset consisted of 152 meshes. To test the precision and accuracy of the laser scanner, 20 colonies previously scanned using a medical CT scanner (House et al. 2018) were rescanned using the laser scanning protocol and morphological measurements were compared (Fig. S1).

\section{Size and shape variables}

For each colony mesh, we calculated two size variables, volume and surface area, and six shape variables that pair off into three axes of shape variation: sphericity and convexity (capturing volume compactness), packing and fractal dimension (capturing surface complexity) and the first moments of area and volume (capturing top-heaviness). Further details of each shape variable follow, but further information and the equations for each variable are contained in Table S2. All variables were calculated in $\mathrm{R}$ (R Core Team 2015), and various functions are included as supplementary material.

The surface area of each mesh was calculated by totalling the area of each triangle in the mesh. Because corals are irregular objects, the volume of each mesh was approximated using the signed volume principal (Zhang and Chen 2001). For each triangle in the mesh, a tetrahedron was formed using the triangle and the origin, and its volume calculated. If the triangle faces away from the origin, its volume is positive, and if the triangle faces towards the origin it is negative. By totalling up the positive and negative volumes, a close approximation to the true volume of the mesh is obtained.

Volume compactness is captured here by two variables. Sphericity $S$ is a size invariant measure of the compactness of an object's volume (Wadell 1935). It is calculated as the ratio of the surface area of a sphere with the same volume as the object and the surface area of the object. Sphericity is bounded by zero (i.e. a theoretical shape that is entirely non-compact, like a plane) and one (i.e. a perfect sphere) and is size independent. Because sphericity is a ratio between zero and one, but never exactly zero or one, it was logit-transformed for analyses. Convexity $C$ is a size invariant measure of the degree to which there is space between different parts of an object (Zunic and Rosin 2004). Convexity is calculated as the volume of an object divided by the volume of its convex hull, where the convex hull is the shape formed by the smallest possible boundary that has no concave areas around an object (Barber et al. 1996). Like sphericity, convexity is bounded by zero (i.e. a theoretical shape that has no volume but some convex volume) and one (i.e. a shape that is entirely convex) and was logit-transformed for analyses.

Surface complexity is also captured by two shape variables. Another form of convexity (which we call packing $P$ for clarity) is a size invariant ratio of how much of an object's surface area is situated internally versus externally in relation to its immediate environment (Zunic and Rosin 2004). It is calculated as the surface area of an object divided by the surface area of its convex hull. A packing value above one indicates that surface area is packed within the volume it occupies (i.e. it is more inverted). Values below one indicate that surface area is more spread out over the volume it occupies (i.e. it is more everted). Convex shapes have a packing equal to one, as the surface area is neither internally nor externally distributed, and objects with an equivalent amount of "inverted" and "everted" surface also have a packing equal to one. As packing is a proportion that can go above or below 1 , it was $\log _{10}$ transformed for analyses. Fractal dimension $D$ captures how surface area fills space and is an estimate of spatial complexity. We calculated fractal dimension using the "cube counting" algorithm, a 3D analogue of the wellknown box counting method (Sarkar and Chaudhuri 1994). Fractal dimension is bounded between two (a plane) and three (a theoretical 2D surface that is completely volume filling) and is size invariant. Fractal dimension is calculated 
as the slope of $\log _{10}(N) \sim \log _{10}(C)$, where $N$ is the total number of cubes that contain any surface of the object and $C$ is the number of equal sized cubes in the $3 \mathrm{D}$ cube array. For each colony, we generated a vector of 25 box sizes that were equally spaced on a $\log _{10}$ scale, with the largest box size having side lengths equal to the longest dimension of the coral. We then selected cube sizes where the smallest was double the size of the laser scanner resolution $\left(0.5 \mathrm{~mm}^{3}\right)$ and the largest was less than the size of longest dimension of the coral. The smaller cut-off was to ensure that boxes that should overlap with a surface, for example a box between two points that are connected, was still counted. The larger cut-off was because the chance of a box with the same size as the coral overlapping a point is 1 , and so every colony would have the same value regardless of their shape.

Top-heaviness was captured by the first moments of volume $\left(V_{\mathrm{VOL}}\right)$ and surface area $\left(V_{\mathrm{AREA}}\right)$ with respect to the vertical distance from the attachment area. For $V_{\text {AREA }}$, the area of each triangle in the mesh was calculated and multiplied by the vertical distance from the centroid of the triangle and the centroid of the attachment plane. For $V_{\mathrm{VOL}}$, a similar algorithm was used. For each triangle in the mesh, a tetrahedron between the triangle and the origin was calculated. The signed volume of each tetrahedron was multiplied by the vertical distance between the centroid of the tetrahedron and the centroid of the attachment plane. Both variables were $\log _{10}$ transformed for analysis. To ensure size invariance, colony meshes were converted to a standard volume of $1 \mathrm{~mm}^{3}$ before these variables were calculated.

\section{Analysis}

We used principal components analysis (PCA) to visualise the morphospace, how growth forms occupied this space, and to identify which shape variables explained most of the variation in colony shape [via the "prcomp" function in R (R Core Team 2015)]. Variables were standardised with a mean of zero and unit variance to reduce the influence of variable scale on the projection. For each principal component, variables were highlighted as important for a given component based on whether their loadings exceeded the null contribution value of $16.6 \%$ (100\% divided by six variables). Pair-wise plots of raw data with Pearson's correlations were used to identify how variables covary and which variables were highly collinear both within a given component and between the variables overall. A LOESS smooth regression was fitted to each variable pair to visualise relationships without making any assumptions about the distributions or linearity of the data.

To test whether shape remained constant with colony size, we used a linear regression approach, with shape variable as the response, and growth form, volume and their interaction as predictors (using the " $1 \mathrm{~m}$ " function in $R$ ). Each shape variable within each growth form was deemed to remain constant with size if zero was within the 95\% confidence intervals for the slope estimate.

To infer whether the morphological variables captured a broad proportion of the subjective variation encoded in growth forms, we first added 95\% confidence ellipses for each growth form to the PCA to visualise how growth forms occupied continuous shape space. We then used multinomial regression to see how well the shape variables could predict the correct growth form [via the "multinom" function from the R package "nnet" (Venables and Ripley 2002)]. We built the initial model based on a set of variables that captured different axes of variation in shape and had low covariance to minimise redundancy of information. No interactions between shape variables were included. Additionally, volume was not included as a main effect as size is not a determining characteristic for growth form. However, volume was included as an interaction effect with shape variables that were shown to vary as a function of volume. Finally, we used this model as the basis for a leave-one-out assessment of predictive accuracy, where a model that omitted an observation was fitted, and the omitted observation data used to generate probabilities of that observation belonging to any one growth form classification, as well as assigning a single class. This was repeated for every observation sequentially and the predicted probabilities and classes were pooled, with the probabilities used for a visual assessment of model performance and the classes used for the generation of a confusion matrix to assess classifier (in this case the multinomial model) performance. The predicted class dataset was assessed via kappa values and balanced accuracy estimates to assess classifier accuracy (Cohen 1960). The overall model was assessed for goodness of fit via McFaddens pseudo- $\mathrm{R}^{2}$.

\section{Results}

\section{Corals in continuous shape space}

$87.5 \%$ of the observed variation in shape was captured by the first two principal components (PC) (Fig. 1). The first PC captured $60 \%$ of the variation across the six-dimensional shape space and was comprised of sphericity, convexity and the first moment of area $\left(V_{\text {AREA }}\right)$. All three variables had contribution values above $16 \%$ with sphericity and $V_{\text {AREA }}$ having joint highest at $25 \%$ and convexity at $22 \%$, suggesting they were all important for explaining the variation along PC1. All three variables were highly correlated (Fig. 2), where sphericity and 


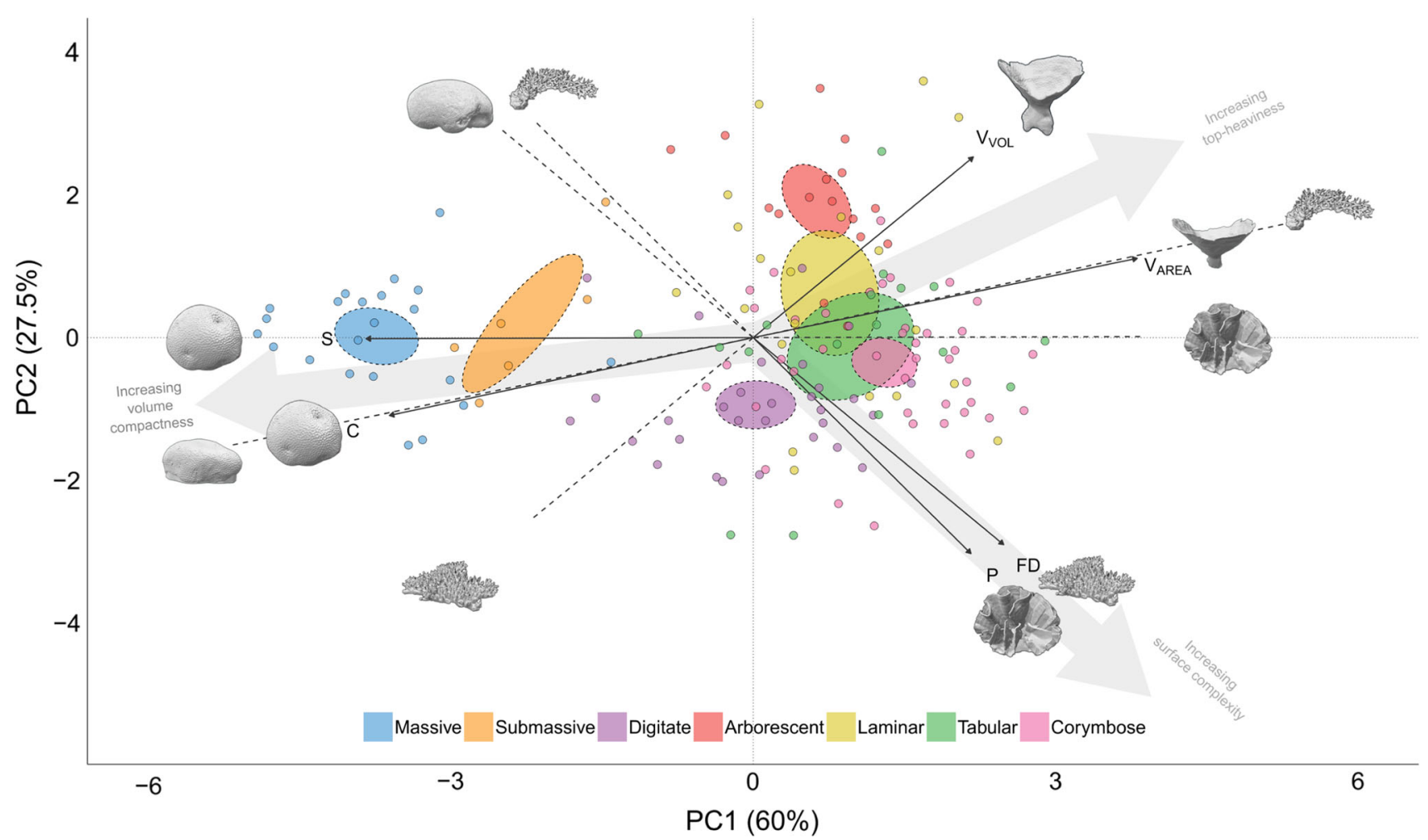

Fig. 1 Projection of 152 coral colonies in two dimensions by the 1st and 2nd principal components (PC) of six-dimensional shape space. Points coloured by growth form classification with $95 \%$ confidence ellipses around the group means. Arrows indicate the loading and direction of each shape variable; $V_{\mathrm{VOL}}=$ first moment of volume $(\mathrm{mm} 4), V_{\text {AREA }}=$ first moment of area $(\mathrm{mm} 3), \mathrm{FD}=$ fractal dimension, $P$ = packing, $C=$ convexity, $S=$ sphericity. The first principal component broadly captures variation in skeletal volume compactness

convexity were positively correlated, with both being negatively correlated with $V_{\text {AREA }}$. Of these three variables, convexity was selected for the predictive model as it had the weakest correlation with the other three variables, therefore minimising redundancy. The second PC captured $27.5 \%$ of the variation and was comprised of packing, fractal dimension and the first moment of volume $\left(V_{\mathrm{VOL}}\right)$. Of these variables, packing had the highest contribution at $35 \%$, followed by fractal dimension $(32 \%)$ and $V_{\mathrm{VOL}}$ (24\%) (Fig. 1). Packing and fractal dimension were highly correlated with each other (Fig. 2); however, both were uncorrelated with $V_{\mathrm{VOL}}$. Of these variables, both $V_{\mathrm{VOL}}$ and packing were selected for the predictive model; $V_{\mathrm{VOL}}$ because it was uncorrelated with the other two variables and packing as it was slightly less correlated with the other variables compared to fractal dimension.

Coral colony shape was constrained by compactness. When sphericity and convexity are high, there was less variation in surface complexity (captured by packing and fractal dimension), with a similar but less pronounced effect on $V_{\text {VOL }}$ (Fig. 2). Additionally, there was a nonlinear
( $\left.S, C \& V_{\text {AREA }}\right)$. The second principal component captures a trade-off between surface area complexity (FD \& $P$ ) and the distribution of volume vertically in the water column $\left(V_{\mathrm{vOL}}\right)$. Images are of the coral specimens that occupy the extremes of each shape variable in the dataset, with some specimens occupying the extreme ends of multiple variables. Larger light grey arrows represent the three morphological axes of variation each represented by two shape variables and have been subjectively added as an aid to help understand the visualisation

decrease in $V_{\text {AREA }}$ as a function of these two variables. Sphericity had the highest correlation scores with the other shape variables. In the PCA projection, we also observed this constraining effect, where the spread of points along PC2 is markedly restricted in extent and density at lower PC1 scores (i.e. higher sphericity and convexity) (Fig. 1). As sphericity and convexity decreased, however, the extent of occupied shape space along PC2 increased.

\section{Growth forms in continuous shape space}

There were two apparent gradients that captured how growth forms were distributed in continuous shape space (Fig. 1). The first was along PC1 where the massive and sub-massive growth forms were isolated from the branching growth forms. The second was along PC2 within the branching group, with the digitate, corymbose, tabular, laminar and arborescent growth forms distributed roughly in that order. The mean position for a given growth form overall was generally constrained within shape space; however at the colony level, many growth forms were 


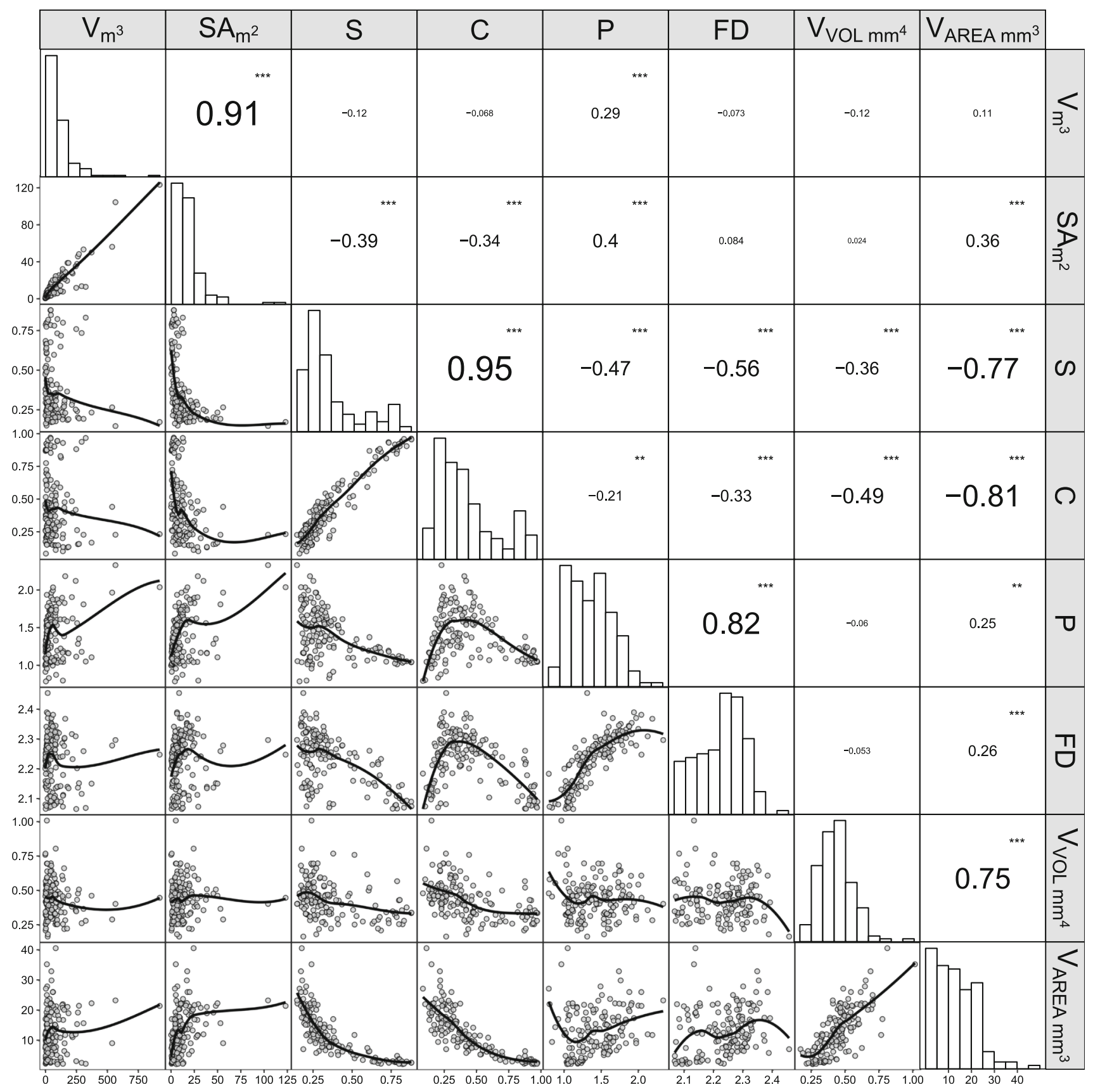

Fig. 2 Pair-plot of six shape and two size variables used in the study. $V=$ volume, $\mathrm{SA}=$ surface area,$S=$ sphericity,$C=$ convexity, $P=$ packing, $\mathrm{FD}=$ fractal dimension, $V_{\mathrm{VOL}}=$ first moment of volume $\left(\mathrm{mm}^{4}\right), V_{\text {AREA }}=$ first moment of area $\left(\mathrm{mm}^{3}\right)$. Bottom triangle

found occupying the same area which was partially explained by variation in shape as a function of size.

\section{Changes in colony shape with size}

While there were no significant correlations between any shape variable and size (represented as colony volume) across all the observed values together except for packing panels: Scatter plots of each variable pair with loess smoother line, $n=152$. Diagonal panels: density plots of each variable, upper triangle panels: Pearson's correlations for each variable pair with significance scores $(* * *=p<0.001, * *=p<0.01)$

(Fig. 2), the shape of a colony did change as a function of size within some growth form and shape variable combinations (Fig. 3). Because each shape variable is size independent (i.e. consistent for the same shape across any range of sizes), the observed changes in shape with size shown here are likely to be genuine differences in shape as size increases. Sphericity decreased with size in the digitate and laminar growth forms. All other growth forms 
Fig. 3 Size by shape variable plot for 152 coral colonies faceted by growth form highlighting changes in shape as a function of colony volume. Panel order from left to right based on average $\mathrm{PC} 1$ values for each growth form. Lines represent linear regression lines with $95 \%$ confidence intervals coloured based on whether the 95\% confidence intervals for slope estimates overlapped with zero. $S=$ sphericity,

$C=$ convexity, $P=$ packing,

$\mathrm{FD}=$ fractal dimension,

$V_{\mathrm{VOL}}=$ first moment of volume,

$V_{\text {AREA }}=$ first moment of surface area

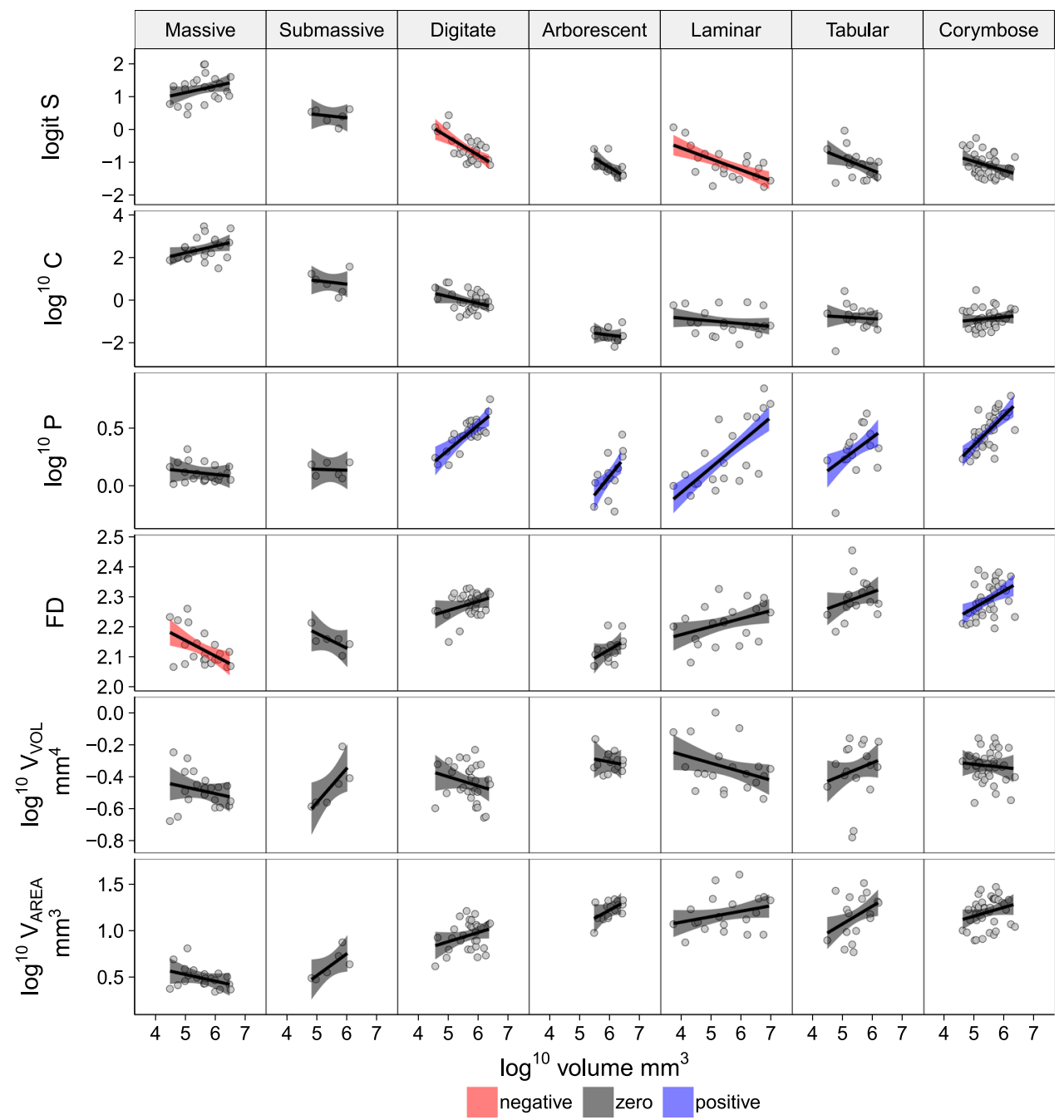

maintained constant sphericity across their observed size range except the tabular and arborescent group which appeared to decrease marginally as volume increased despite their slope estimate confidence intervals overlapping with zero (Fig. 3). Packing increased with size fastest in the arborescent group, followed by the corymbose, laminar, digitate and tabular growth forms, with the massive and sub-massive colonies remaining constant. The massive group decreased in fractal dimension with size and the corymbose colonies increased with size. All the other variables remained constant as a function of volume based on the $95 \%$ confidence intervals. However, there was some evidence to suggest that both $V_{\mathrm{VOL}}$ and $V_{\mathrm{AREA}}$ may scale with size in some growth forms (Fig. 3).

\section{Capturing qualitative growth forms using quantitative variables}

Growth form was correctly predicted by four shape variables in conjunction with volume (Fig. 4). The model included convexity, packing, $V_{\mathrm{VOL}}$ and fractal dimension, with interaction terms between volume and both packing and fractal dimension. The final model explained $74 \%$ of the deviance (McFadden's pseudo $R^{2}$ of 0.62 , d.f $=42$ ). Overall, the model predicted growth forms with a high degree of accuracy (kappa $=0.66$ ). The growth forms in order of highest to lowest balanced accuracy were: massive (95.1\%, $n=22)$, arborescent $(92.6 \%, n=16)$, sub-massive $(91.3 \%, n=6)$, digitate $(81 \%, n=30)$, corymbose $(80.7 \%$, $n=41)$, tabular $(77.2 \%, n=17)$ and laminar $(70.2 \%$, $n=20)$. Fractal dimension was added to the model as the three shape variables alone were unable to distinguish between tabular and laminar growth forms, despite the balanced accuracy of all other groups being above $79 \%$. The probability of the correct class in the final model was the highest for all growth forms and was distinct from all other potential classes (Fig. 4). The massive group had the highest mean probability $(0.95 \pm 0.04)$, with the laminar and tabular having the lowest $(0.48 \pm 0.07$ and $0.50 \pm 0.1$, respectively). 


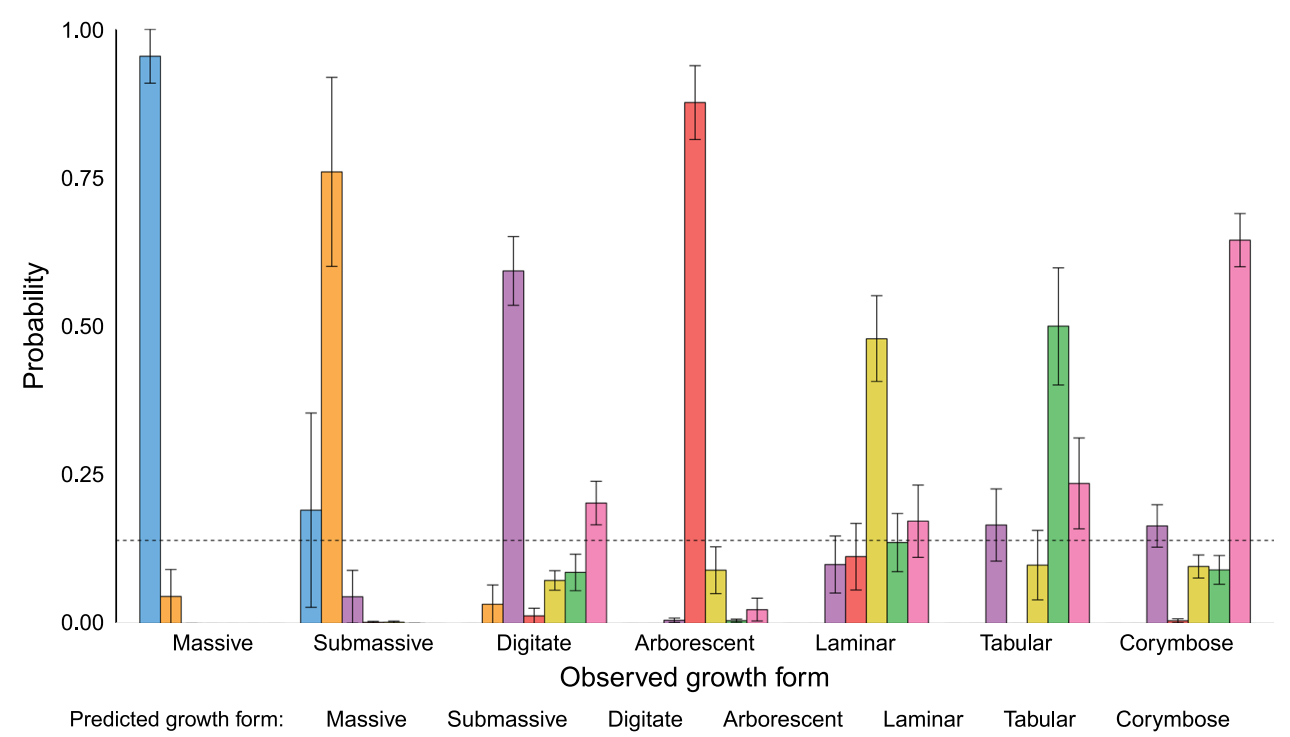

Fig. 4 Observed growth form by predicted growth form probabilities for seven coral growth forms based on a multinomial regression using continuous shape variables. Data generated via a leave-one-out approach, where each observation was left out of the initial model and classification probabilities generated for the missing observation, repeated for each observation in the dataset. Coloured bars represent

\section{Discussion}

We developed six quantitative shape variables and showed that variation in volume compactness, surface complexity and top-heaviness explained much of the variation in coral shape. The observed changes in some variables with colony size resulted in colonies that straddled traditional growth form classifications (Fig. 3). We found that four morphological variables can predict traditional growth form classifications with accuracies ranging from 70 to 95\%, demonstrating that these variables captured most of the variation encoded in traditional growth form classifications (Fig. 4). Our approach was able to place coral colony morphology along continuous axes of functional variation without relying on homologous structures or landmarks, providing a set of morphological traits to explore general links between shape and biological and ecological processes across multiple growth forms and colonies simultaneously.

Coral morphology was partitioned into three main axes of variation (Figs. 1, 2). Variation in volume compactness captured a gradient from non-branching to highly branching colonies. However, compactness constrained surface complexity and top-heaviness, where colonies with higher levels of compactness tended to be smooth and bottom heavy. Furthermore, each of the three axes can provide causal explanations for biological processes. For example, volume compactness may be a suitable trait for explaining why more massive morphologies have less variable and average probability of being classified as a given growth form with standard errors. Horizontal dashed line represents the unbalanced expected probability if all classes were randomly assigned (100\%/7 possible classes) and was used to determine which incorrectly predicted classes were significantly misclassified for each growth form. $n=152$

slower overall growth because it captures a gradient from massive to more complex forms and relates to surface areato-volume ratios (Dornelas et al. 2017). Similarly, variation in top-heaviness, capturing a gradient from lower lying to tabular colonies, may be a trait that can test ideas related to benthic competition strategies (e.g. lateral benthic expansion vs indirect shading and competitive escape) (Jackson 1979). Variation in surface complexity is related to competition and resource use, where colonies with their surfaces distributed in a complex way have less resources (e.g. light, nutrients) per unit surface area but can have more polyps packed within a given space (Wangpraseurt et al. 2012). For example, if light levels are low, uniformly spreading out surface area maximises incoming light per surface area, which is reflected by increasing abundance of plating colonies as depth increases (Chappell 1980). These hypotheses are based on organism performance, but others can be formulated across a range of scales. Examples include low compactness colonies providing habitat for juvenile fishes (Alvarez-Filip et al. 2011), high compactness colonies increasing reef framework building (Rasser and Riegl 2002), high surface complexity increasing larval recruitment (Hata et al. 2017) and niche diversification being increased by top-heavy, tabular colonies (Kerry and Bellwood 2015). The ability to formulate and test these types of causal hypotheses offers a direct approach for linking form to function not possible using growth forms, and not clouded by metrics that conflate shape and size. 
Growth forms typically occupied specific areas of continuous shape space, but the large amount of overlap between them suggests that growth forms are less distinct than their discrete nature implies (Figs. 1, 3). Growth forms were also not distributed along a single trajectory of morphological variation which highlights that morphological variation between growth forms occurs along multiple axes. Therefore, a single ordinal classification of categories, for example from most to least "complex", would be misguided. Overall, the semi-distinct, semi-overlaying distribution and variation in growth forms at once confirms that growth forms work as morphologically distinct classifications to some degree but at the same time suggests that they are less definite and defined than implied by the nature of assigning a single category. This potentially unsatisfactory statement is made more palatable once morphological plasticity and the observed changes in shape as a function of size within growth form (Fig. 3) are considered: not every corymbose colony looks alike in the same way that each member of a wildebeest herd does, and a tabular colony only looks truly tabular after an initial period of growth up and out from the benthos. As such, the size distribution of colonies may have implications for the shape distributions of colonies within a growth form. Within growth form changes in life history traits with size have been highlighted previously (Madin et al. 2014; Álvarez-Noriega et al. 2016; Dornelas et al. 2017), which may be partially explained by ontogenetic changes in morphology and morphology-related processes. While variation in processes between growth forms acts as an indicator that morphology plays an important role, the incomplete and overtly definite nature of growth form categories are unsuitable for establishing causal links. The shape variables proposed here, however, offer an approach for establishing such links due to their quantitative, nondiscrete nature.

While this study included a wide range of growth forms and sizes, there are unobserved sources of variation that may fill in or stretch the boundaries of the observed shape space if added. Encrusting colonies, which extend laterally over the surrounding substrate, were not included due to the difficult nature of obtaining whole colony specimens and the fact that the three-dimensional shape of an encrusting colony is contingent on the local substrate it encrusts, although in situ measurements of encrusting forms should be possible via photogrammetry techniques. Columnar colonies are also absent due to a lack of intact specimens in the museum collections. The less populated area of the observed morphospace between the massive and sub-massive growth forms and the remaining growth forms would likely be occupied by columnar colonies given their semi-sturdy, semi-branching shape. While the range in colony size in the study varied over three orders of magnitude, including both smaller and larger colonies in the dataset may also further fill in and expand the observed shape space. Further, there are likely to be microstructural differences between live colonies and skeletons; however, the focus of this study was on broader differences at the colony scale which are likely to be larger than differences between live and dead colony scans.

Both the approach and results of our study have applications for relating morphology to process in other taxa. Because the variables used in this study require no taxonspecific information to calculate (e.g. landmarks) they can be used to measure and compare morphological variation across any organism where a suitable 3D representation is available. Other colonial organisms such as sponges, soft corals, gorgonians and macroalgae are similar in their range of geometric complexity and are exposed to similar conditions, which suggest that similar trade-off axes should exist in these taxa. Measuring complex colony shapes across taxonomic groups would allow for empirical testing of the theoretical work on morphological strategies laid out by Jackson (1979). In the terrestrial realm, there is a large body of work on the functional ecology of plants which partially overlaps with corals given that both groups are sessile, able to experience partial mortality, and have a photosynthetic component. Going a step further, it should also be possible to compare morphology across a range of organisms, from bacteria to blue whales, to potentially uncover universal drivers of morphological adaptations via the variables outlined in this study.

This study provides a comprehensive set of traits that partition shared and unique variation between growth forms and highlight size-dependent changes in shape within growth forms. These traits have strong theoretical links to many processes important for both corals and their roles as ecosystem engineers and allow for causal explanations of phenomena to be established across a broad range of morphological variation. This study provides an empirical toolkit and theoretical backbone for future reef research that is timely given the ongoing work on threedimensional metrics and methods, and the need to establish a broader understanding of how morphology maps to function across scales.

Acknowledgements We would like to acknowledge Francois Leclerc and CREAFORM Inc. for providing equipment and technical support for the data collection. Luc Bidaut and Jenny House for CT scan data for calibration. Miranda Lowe and the Natural History Museum of London, Tom Bridge and the Museum of Tropical Queensland, and the Bell Pettigrew museum for facilitating specimen access and data collection. Kristin Precoda, Rachael Woods and Damaris Torrez-Pulliza for proofreading and feedback throughout. MD was supported by the John Templeton Foundation (60501) and a Leverhulme Fellowship, while JM was supported by the Australian Research Council (FT110100609) during the period this research was undertaken. 
Data availability Morphological traits data available from figshare. https://figshare.com/articles/CoralColonyMorphologyData_Whole Colonies_MediumToHighQuality_LaserScanned/8115674. Scripts and functions available from https://github.com/Kylezx 1/ QuantifyingCoralMorphology.

\section{Complains with ethical standard}

Conflict of interest The authors have no competing interests to declare.

Open Access This article is distributed under the terms of the Creative Commons Attribution 4.0 International License (http://crea tivecommons.org/licenses/by/4.0/), which permits unrestricted use, distribution, and reproduction in any medium, provided you give appropriate credit to the original author(s) and the source, provide a link to the Creative Commons license, and indicate if changes were made.

\section{References}

Almany GR (2004) Differential effects of habitat complexity, predators and competitors on abundance of juvenile and adult coral reef fishes. Oecologia 141:105-113

Alvarez-Filip L, Gill JA, Dulvy NK (2011) Complex reef architecture supports more small-bodied fishes and longer food chains on Caribbean reefs. Ecosphere 2:1-17

Álvarez-Noriega M, Baird AH, Dornelas M, Madin JS, Cumbo VR, Connolly SR (2016) Fecundity and the demographic strategies of coral morphologies. Ecology 97:3485-3493

Baird AH, Marshall PA (2002) Mortality, growth and reproduction in Scleractinian corals following bleaching on the great barrier reef. Mar Ecol Prog Ser 237:133-141

Barber CB, Dobkin DP, Huhdanpaa H (1996) The quickhull algorithm for convex Hulls. ACM Trans Math Softw 22:469-483

Bell J, Galzin R (1984) Influence of live coral cover on coral-reef fish communities. Mar Ecol Prog Ser 15:265-274

Bishop JDD (1989) Colony form and the exploitation of spatial refuges by encrusting Bryozoa. Biol Rev 64:197-218

Bruno JF, Edmunds PJ (1997) Clonal variation for phenotypic plasticity in the coral Madracis Mirabilis. Ecology 78:2177-2190

Bythell J, Pan P, Lee J (2001) Three-dimensional morphometric measurements of reef corals using underwater photogrammetry techniques. Coral Reefs 20:193-199

Zhang C, Chen $T$ (2001) Efficient feature extraction for 2D/3D objects in mesh representation. In: 2001 international conference on image processing 2:935-938

Chappell J (1980) Coral morphology, diversity and reef growth. Nature 286:249-252

Cohen J (1960) A coefficient of agreement for nominal scales. Educ Psychol Meas 20:37-46

Connell JH, Hughes TP, Wallace CC, Tanner JE, Harms KE, Kerr AM (2004) A long-term study of competition and diversity of corals. Ecol Monogr 74:179-210

Denny MW (1993) Air and water: the biology and physics of life's media. Princeton University Press, Princeton

Done T (2011) Corals: environmental controls on growth. In: Hopely D (ed) Encyclopedia of modern coral reefs: structure, form and process. Springer, Dordrecht, pp 281-293

Dornelas M, Madin JS, Baird AH, Connolly SR (2017) Allometric growth in reef-building corals. Proc R Soc Lond B Biol Sci 284:20170053
Filatov MV, Frade PR, Bak RPM, Vermeij MJA, Kaandorp JA (2013) Comparison between colony morphology and molecular phylogeny in the Caribbean Scleractinian coral genus Madracis. PLOS One 8:e71287

Filatov MV, Kaandorp JA, Postma M, van Liere R, Kruszyński KJ, Vermeij MJA, Streekstra GJ, Bak RPM (2010) A comparison between coral colonies of the genus Madracis and simulated forms. Proc R Soc Lond B Biol Sci 277:3555-3561

Foster AB (1979) Phenotypic plasticity in the reef corals Montastraea annularis (Ellis \& Solander) and Siderastrea siderea (Ellis \& Solander). J Exp Mar Biol Ecol 39:25-54

Friedlander AM, Parrish JD (1998) Habitat characteristics affecting fish assemblages on a Hawaiian coral reef. J Exp Mar Biol Ecol 224:1-30

Gladfelter EH, Monahan RK, Gladfelter WB (1978) Growth rates of five reef-building corals in the Northeastern Caribbean. Bull Mar Sci 28:728-734

Glynn PW, Manzello DP (2015) Bioerosion and coral reef growth: a dynamic balance. Coral reefs in the anthropocene. Springer, Dordrecht, pp 67-97

Graham NAJ, Nash KL (2013) The importance of structural complexity in coral reef ecosystems. Coral Reefs 32:315-326

Hata T, Madin JS, Cumbo VR, Denny M, Figueiredo J, Harii S, Thomas CJ, Baird AH (2017) Coral larvae are poor swimmers and require fine-scale reef structure to settle. Sci Rep 7:2249

Hoegh-Guldberg O (1988) A method for determining the surface area of corals. Coral Reefs 7:113-116

Hoogenboom MO, Connolly SR, Anthony KRN (2008) Interactions between morphological and physiological plasticity optimize energy acquisition in corals. Ecology 89:1144-1154

House JE, Brambilla V, Bidaut LM, Christie AP, Pizarro O, Madin JS, Dornelas M (2018) Moving to 3D: relationships between coral planar area, surface area and volume. PeerJ 6:e4280

Jackson JBC (1977) Competition on marine hard substrata: the adaptive significance of solitary and colonial strategies. Am Nat 111:743-767

Jackson JBC (1979) Morphological strategies of sessile animals. In: Larwood G, Rosen BR (eds) Biology and systematics of colonial animals. Academic Press, New York, pp 499-555

Johannes RE, Wiebe WJ (1970) Method for determination of coral tissue biomass and composition. Limnol Oceanogr 15:822-824

Kaandorp JA, Lowe CP, Frenkel D, Sloot PMA (1996) Effect of nutrient diffusion and flow on coral morphology. Phys Rev Lett 77:2328-2331

Karlson RH (1986) Disturbance, colonial fragmentation, and sizedependent life history variation in two coral reef cnidarians. Mar Ecol Prog Ser 28:245-249

Kerry JT, Bellwood DR (2012) The effect of coral morphology on shelter selection by coral reef fishes. Coral Reefs 31:415-424

Kerry JT, Bellwood DR (2015) Do tabular corals constitute keystone structures for fishes on coral reefs? Coral Reefs 34:41-50

Koehl MA (1999) Ecological biomechanics of benthic organisms: life history, mechanical design and temporal patterns of mechanical stress. J Exp Biol 202:3469-3476

Lavy A, Eyal G, Neal B, Keren R, Loya Y, Ilan M (2015) A quick, easy and non-intrusive method for underwater volume and surface area evaluation of benthic organisms by $3 \mathrm{D}$ computer modelling. Methods Ecol Evol 6:521-531

Lirman D (2000) Fragmentation in the branching coral Acropora palmata (Lamarck): growth, survivorship, and reproduction of colonies and fragments. J Exp Mar Biol Ecol 251:41-57

Madin JS (2005) Mechanical limitations of reef corals during hydrodynamic disturbances. Coral Reefs 24:630-635

Madin JS, Baird AH, Dornelas M, Connolly SR (2014) Mechanical vulnerability explains size-dependent mortality of reef corals. Ecol Lett 17:1008-1015 
Meesters EH, Wesseling I, Bak RPM (1996) Partial mortality in three species of reef-building corals and the relation with colony morphology. Bull Mar Sci 58:838-852

Nash KL, Allen CR, Barichievy C, Nyström M, Sundstrom S, Graham NAJ (2014) Habitat structure and body size distributions: crossecosystem comparison for taxa with determinate and indeterminate growth. Oikos 123:971-983

Naumann MS, Niggl W, Laforsch C, Glaser C, Wild C (2009) Coral surface area quantification-evaluation of established techniques by comparison with computer tomography. Coral Reefs 28:109-117

R Core Team (2015) R: a language and environment for statistical computing. R Foundation for Statistical Computing, Vienna

Rasser M, Riegl B (2002) Holocene coral reef rubble and its binding agents. Coral Reefs 21:57-72

Reichert J, Backes AR, Schubert P, Wilke T (2017) The power of 3D fractal dimensions for comparative shape and structural complexity analyses of irregularly shaped organisms. Methods Ecol Evol 00:1-9

Richardson LE, Graham NAJ, Hoey AS (2017a) Cross-scale habitat structure driven by coral species composition on tropical reefs. Sci Rep 7:7557

Richardson LE, Graham NAJ, Pratchett MS, Hoey AS (2017b) Structural complexity mediates functional structure of reef fish assemblages among coral habitats. Environ Biol Fishes 100:193-207

Sarkar N, Chaudhuri BB (1994) An efficient differential box-counting approach to compute fractal dimension of image. IEEE Transactions on Systems, Man, and Cybernetics 24:115-120
Sebens KP (1987) The ecology of indeterminate growth in animals. Annu Rev Ecol Syst 18:371-407

Shaish L, Abelson A, Rinkevich B (2007) How plastic can phenotypic plasticity be? The branching coral Stylophora pistillata as a model system. PLOS One 2:e644

Shapiro JA (1995) The significances of bacterial colony patterns. BioEssays 17:597-607

Veron JEN (2000) Corals of the world. Australian Institute of Marine Science, Townsville

Veron JEN (2002) New species described in corals of the world. Australian Institute of Marine Science, Townsville

Vogel S (1996) Life in moving fluids: the physical biology of flow. Princeton University Press, Princeton

Venables WN, Ripley BD (2002) Modern applied statistics with s. Spinger, New York

Wadell H (1935) Volume, shape, and roundness of quartz particles. J Geol 43:250-280

Wangpraseurt D, Larkum AW, Ralph PJ, Kühl M (2012) Light gradients and optical microniches in coral tissues. Front Microbiol 3:316

Zunic J, Rosin PL (2004) A new convexity measure for polygons. IEEE Trans Pattern Anal Mach Intell 26:923-934

Publisher's Note Springer Nature remains neutral with regard to jurisdictional claims in published maps and institutional affiliations. 